\section{$\underset{\text { hommes }}{\text { \& migrations }}$}

\section{Hommes \& migrations}

Revue française de référence sur les dynamiques

migratoires

\section{$1291 \mid 2011$}

Diasporas sri lankaises

\title{
Le temple et les défunts
}

Religion et reconstruction identitaire chez les Tamouls hindous d'origine sri lankaise à Montréal

\section{Mark Bradley et Pierre-Yves Trouillet}

\section{OpenEdition}

\section{Journals}

Édition électronique

URL : http://journals.openedition.org/hommesmigrations/687

DOI : 10.4000/hommesmigrations.687

ISSN : 2262-3353

\section{Éditeur}

Musée national de l'histoire de l'immigration

\section{Édition imprimée}

Date de publication : 1 mai 2011

Pagination : 116-127

ISSN : 1142-852X

Référence électronique

Mark Bradley et Pierre-Yves Trouillet, « Le temple et les défunts », Hommes \& migrations [En ligne],

1291 | 2011, mis en ligne le 31 décembre 2013, consulté le 21 avril 2019. URL : http://

journals.openedition.org/hommesmigrations/687 ; DOI : 10.4000/hommesmigrations.687 


\section{Le temple et les défunts Religion et reconstruction identitaire chez les Tamouls hindous d’origine sri lankaise à Montréal}

Par Mark Bradley, doctorant en sciences des religions, université du Québec à Montréal, et Pierre-Yves Trouillet, post-doctorant en géographie, UMR ADES (CNRS-université de Bordeaux)

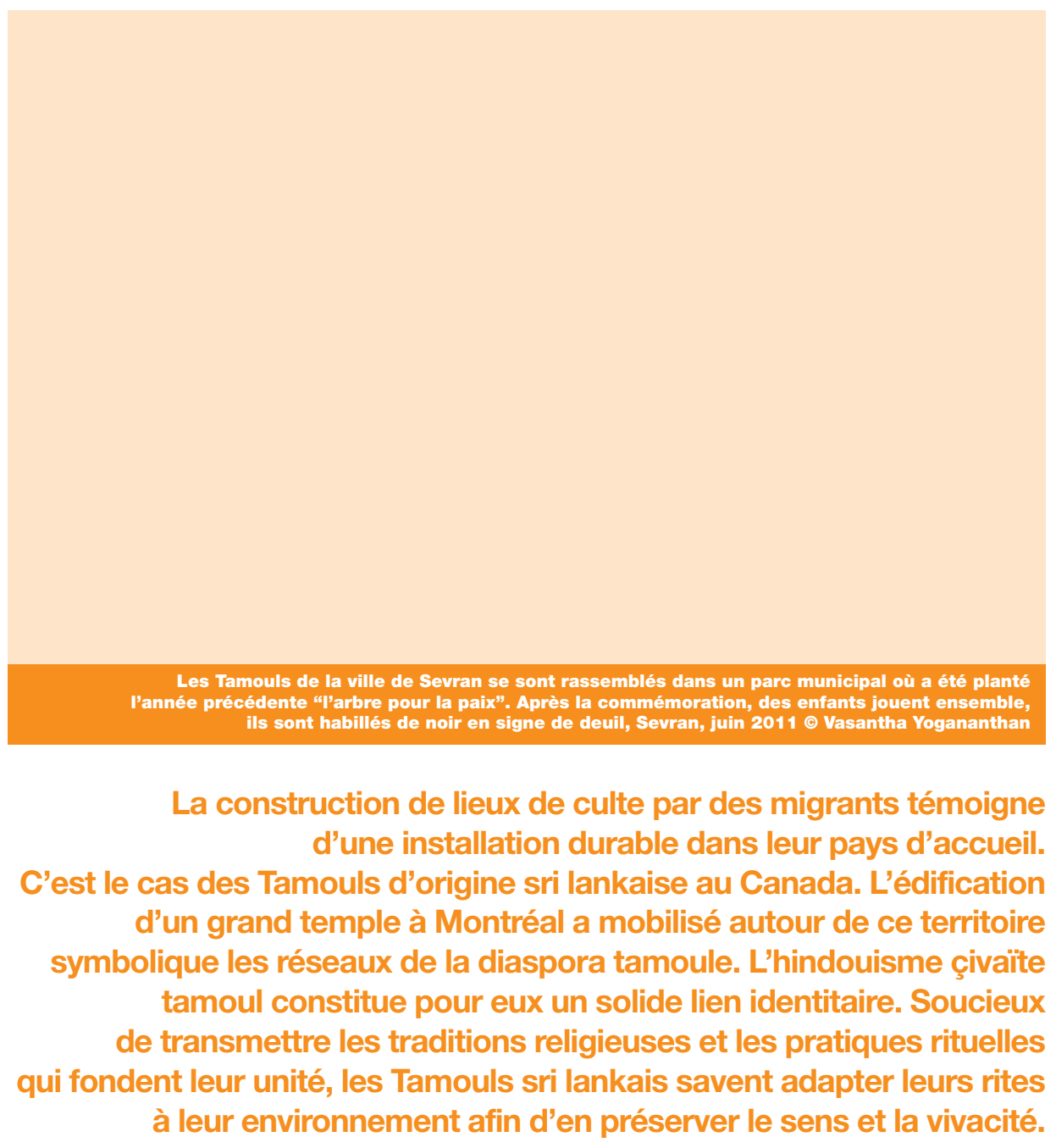


L'émigration tamoule de Sri Lanka vers le Canada débuta dès après le "Juillet noir" de 1983 au cours duquel des milliers de Tamouls furent assassinés, et plus de 150000 d'entre eux durent fuir après avoir tout perdu : maison, commerce, terre, $\operatorname{etc}^{(1)}$. Nombreux furent alors celles et ceux qui demandèrent le statut de réfugié auprès d'ambassades étrangères. Le Canada fut particulièrement généreux, acceptant sur son territoire plusieurs dizaines de milliers de réfugiés.

Les premiers arrivants s'installèrent au Québec, première province à accorder des avantages sociaux aux réfugiés. Cette politique fut ensuite appliquée en 1985 en Ontario, devenu depuis la principale destination des Tamouls sri lankais immigrant au Canada. D'après les chiffres du dernier recensement, 138000 tamoulophones résidaient au Canada en 2006. Il est néanmoins fort probable que ces chiffres soient très en deçà de la réalité et que le Canada compte plusieurs centaines de milliers de Tamouls d'origine sri lankaise sur son territoire.

Lorsqu'un réfugié entreprend de s'intégrer à sa société d'accueil, il est confronté, en même temps qu'à des impératifs de survie, à un difficile processus de reconstruction identitaire ${ }^{(2)}$. Il doit se redéfinir en relation avec ce nouveau milieu, mais aussi par rapport à son passé et à tous les signifiants qui y sont liés. Or, au nombre des marqueurs identitaires les plus puissants, on note la langue et l'alimentation, mais aussi la religion ${ }^{(3)}$. Aussi cet article propose-t-il d'étudier certaines facettes de la reconstruction identitaire des Tamouls hindous d'origine sri lankaise à Montréal en interrogeant le champ religieux.

La religion occupe depuis des siècles une place majeure dans la vie sociale, existentielle et culturelle des Tamouls et offre ainsi un angle d'analyse précieux pour approcher les modalités de reconstruction de l'identité tamoule en situation de diaspora. Il est ici question de l'hindouisme, et plus précisément de l'hindouisme çivaïte tamoul, fortement imprégné de la philosophie du Saiva Siddhanta qui reste la religion majoritaire des Tamouls de Sri Lanka, de l'Inde du Sud et de la diaspora. L'article repose sur deux thèmes d'analyse complémentaires, mais dont les essences sont opposables d'un point de vue religieux, car souvent opposées dans la culture hindoue en général et tamoule en particulier. Il s'agit, d'une part, du temple, lieu saint par excellence et de rencontre avec la divinité, et, d'autre part, des rites funéraires, associés au monde "inauspicieux" de la mort et de la "pollution" rituelle. Le choix de réunir ici ces deux objets d'étude, le temple et le défunt, est motivé parce que l'un comme l'autre contribuent à l'entretien d'un univers de sens ${ }^{(4)}$ dont l'importance est d'autant plus grande en contexte diasporique ${ }^{(5)}$. Aussi, malgré leur incompatibilité rituelle, proposons-nous d'étudier ici le temple et les rites funéraires, en raison de leur place centrale dans et pour cet univers de sens. 


\section{Le temple de Murugan de Montréal}

Depuis près de deux mille ans, le temple est une véritable institution pour les sociétés tamoules ${ }^{(6)}$. Outre son rôle premier de lieu de culte, le temple est doté, en Inde comme à Sri Lanka, de fonctions socio-symboliques, économiques et politiques majeures. Aussi, en construisant un temple dans son pays d'accueil, une communauté tamoule en situation de diaspora bâtit-elle non seulement un lieu religieux, mais également un des principaux éléments constitutifs de son identité.

Le temple dont il est ici question, le Montreal Arulmigu Thiru Murugan Temple, est situé dans la banlieue montréalaise de Dollard-des-Ormeaux. L'édifice est dédié à la divinité Murugan (ou Murukan), connue comme l'un des fils du grand dieu Siva et comme le frère de Ganesa, le célèbre dieu à tête d'éléphant. Ce temple est loin d'être le seul lieu de culte hindou du Canada - qui en compte plus d'une soixantaine aujourd'hui -, mais il présente l'intérêt d'être le premier du pays à avoir été fondé par une communauté exclusivement tamoule sri lankaise. Ce cas d'étude permet donc de voir mieux que tout autre comment, dans le contexte socio-culturel tamoul, la fondation d'un temple en diaspora participe à la reconstruction d'une identité déracinée.

\section{La construction d'un lieu identitaire}

La construction d'un temple nécessite tout d'abord la (re)constitution d'une communauté religieuse locale. Pour le cas du temple Thiru Murugan, ce regroupement communautaire fut très rapide. Dès 1985, un groupe de Tamouls çivaïtes originaires de Sri Lanka fonda la Mission Saiva du Québec, une organisation religieuse à but non lucratif dont l'objectif était de bâtir un temple tamoul "authentique" à Montréal, reprenant le style des temples de Jaffna, qui "suivent les Saiva-Agama" culturels de la communautét ${ }^{(8) "}$. Cette rapidité dans la décision de fonder un temple en terre étrangère témoigne de la conscience déjà partagée, parmi ces réfugiés tamouls à peine arrivés à Montréal, des difficultés qu'ils auraient à retourner à Sri Lanka et de la durabilité de leur installation au Canada. Car bâtir un temple pour la communauté locale, ancrer les dieux dans le sol et faire venir des prêtres, c'est finalement produire un haut lieu sur ce qui deviendra un nouveau territoire. Sept ans après sa création, la Mission aiva du Québec parvint à acheter un terrain de deux acres dans la ville de Dollard-des-Ormeaux, en banlieue ouest de Montréal.

Bien que ce temple soit ouvert à toutes les communautés, il fut le premier du Canada à ne compter que des tamoulophones d'origine sri lankaise au sein de son comité de gestion. Ce mode d'organisation permet aux Tamouls d'éviter les querelles intercommunautaires pouvant apparaître entre des gestionnaires originaires de différentes 
régions d'Asie du Sud et qui ne suivent pas exactement les mêmes traditions. Cette gestion du temple assure ainsi une meilleure cohésion identitaire à la communauté tamoule et facilite la mise en place d'activités socio-culturelles annexes proprement tamoules, comme l'enseignement linguistique et artistique.

En 2008, les membres du comité de gestion présentaient ce temple comme le seul de tout le pays à être orné d'une grande tour sculptée (tam. raca-kopuram) orientée à l'est. Cette exclusivité témoignait également de son mode de construction fidèle aux normes religieuses et architecturales fondées sur les Agama çivaïtes tamouls, ce qui était une nouveauté pour l'époque au Canada. Le temple Thiru Murugan fut aussi le premier au Québec à mobiliser un architecte (tam. stapati) et des artisans (tam. cirpi) venus de l'étranger et spécialisés dans la construction des temples "agamiques". Outre le prestige et la fierté identitaire que la construction d'un temple aussi monumental et aussi respectueux de l'orthodoxie agamique pouvait procurer à la communauté

Dès ses origines, le temple de Murugan de Montréal avait donc vocation à être plus tamoul que les autres temples du Canada, et ce tant par la communauté qu'il servirait que par l'orthodoxie qui le fonderait et par la divinité qu'il hébergerait. tamoule de Montréal, cette volonté de construire un tel temple témoigne également de leur quête d'authenticité, apparaissant comme nécessaire à la reconstruction de leur identité religieuse (ce qui se retrouve également pour les rites funéraires). Ce souci d'authenticité illustre à nouveau le processus d'ancrage des Tamouls sri lankais au Canada, car pourquoi se donner autant de mal à reproduire si fidèlement une tradition religieuse dans le pays d'accueil si ce n'est pour y demeurer?

Conjointement à l'authenticité, la distinction est un autre phénomène majeur à l'ceuvre dans la reconstruction identitaire des Tamouls qui s'est opérée à travers la construction du temple de Murugan à Montréal. Nous avons vu que la volonté de distinction communautaire proprement tamoule est apparue dès la fondation de la Mission Saiva du Québec en 1985 et qu'elle se retrouve également dans le mode de gestion du temple. Or celle-ci est également signifiée par le choix de la divinité tutélaire du temple, Murugan, dont la relation privilégiée avec l'identité tamoule est bien connue, en Inde comme dans la diaspora ${ }^{(9)}$. Une brochure du temple présente ainsi cette divinité comme "le dieu préféré" des Tamouls ${ }^{(10)}$ et l'un des membres du comité de gestion expliqua ce choix de divinité par le fait que "Murugan est un dieu tamoul" et parce que les Tamouls sri lankais sont "des dévots [bhakta] du Seigneur Murugan".

Dès ses origines, le temple de Murugan de Montréal avait donc vocation à être plus tamoul que les autres temples du Canada, et ce tant par la communauté qu'il servirait 
que par l'orthodoxie qui le fonderait et par la divinité qu'il hébergerait. En effet, la communauté en question, l'orthodoxie prônée et la divinité choisie avaient pour premier point commun d'être spécifiquement tamoules. Avec ce temple, il était donc bien question de reconstruire non seulement un lieu de culte pour la communauté, mais aussi une identité tamoule se voulant distincte et authentique.

\section{Un produit diasporique}

De nos jours, les temples bâtis par la diaspora hindoue ne doivent pas être considérés comme des fragments isolés. Il s'agit au contraire de lieux souvent interconnectés par le biais de réseaux socio-religieux de dimensions locale, nationale et transnationale. Le temple de Murugan de Montréal, dont la construction fut achevée en 2006, ne fait pas exception. Il montre à l'inverse comment des logiques transnationales peuvent se mêler aux stratégies d'une communauté locale, afin de permettre la réalisation d'un temple diasporique.

Pour que ce temple "agamique" soit édifié, il fallait tout d'abord recourir à un prêtre ayant les compétences requises en astrologie (skt. vastusastra) et une connaissance approfondie des Agama. Ce rôle fut assuré par le prêtre en chef (tam. kurukkal) du temple, un officiant çivaïte érudit de caste brahmane (sivacarya) originaire de Sri Lanka. La construction du bâtiment fut quant à elle supervisée par l'architecte Sthapathy K. Dakshinamoorthy et réalisée par douze artisans, tous venus spécialement du Tamil Nadu (le " pays tamoul") en Inde du Sud. Puis, pour célébrer la consécration rituelle (tam. makakumpapisekam) du temple, une trentaine de prêtres tamouls se sont déplacés d'autres villes du Canada, des États-Unis et même d'Europe et d'Asie, où ils étaient employés, afin d'assister le prêtre en chef.Enfin, et plus largement, la construction du temple a reçu le soutien d'une vingtaine d'organisations religieuses tamoules basées elles aussi au Canada, à Sri Lanka (district de Jaffna), au Tamil Nadu et aux États-Unis, mais également en Malaisie, au Royaume-Uni, en Australie et en Nouvelle-Zélande. Les pôles diasporiques qui émergent de cette série d'acteurs religieux indiquent que la construction du temple de Murugan de Montréal a mobilisé un réseau assez représentatif - bien que non exhaustif - de la géographie transnationale de la diaspora tamoule. Notons également que trois niveaux d'échelle apparaissent et s'entrecroisent dans ce fonctionnement réticulaire : le niveau local, avec la Mission Saiva du Québec fondatrice du temple ; le niveau national, représenté par les prêtres et les comités de gestion des autres temples du Canada ; et le niveau transnational, incarné par les acteurs installés dans des territoires plus lointains.

Ainsi, au travers des objectifs qui le fondent et des acteurs qui lui ont donné vie, le temple de Murugan de Montréal illustre à merveille combien l'identité diasporique peut être "simultanément locale et globale", et en quoi "le processus diasporique intègre 
(...) territorialisation et fonctionnement en réseau ${ }^{(11)}$ ". Bien que ce temple soit archétypique, et que tous les temples tamouls du Canada n'aient pas bénéficié de la participation d'acteurs aussi nombreux, cet exemple démontre toute l'importance des réseaux transnationaux dans la reconstruction des identités déterritorialisées.

\section{Le rituel funéraire çivaïte de Sri Lanka à Montréal}

Pour les Tamouls hindous, la mort est un passage, une étape dans le cycle du samsara, mais aussi l'occasion d'un samskara, un rite de perfectionnement. La mort met un terme à une existence (tam. immai), et les survivants doivent profiter de l'état intermédiaire dans lequel se trouve l'âme (tam. attumam, skt. atman) du défunt pour l'honorer et la mettre en valeur de façon que celle-ci jouisse du meilleur sort possible lors de sa prochaine existence (tam. marumai) $)^{(12)}$.

\section{La préparation du corps et l'hommage rendu au défunt}

À Sri Lanka, le plus souvent, les gens meurent à la maison, au milieu des leurs, et ce sont eux qui procèdent à la toilette du corps. Dans la maison en deuil, on ne fait plus jouer ni télé ni musique, les portraits de famille sont retournés contre le mur, les feux de cuisine sont éteints et toute nourriture est désormais apportée de l'extérieur. Cette étape dure un ou deux jours, le temps pour la famille étendue de se réunir. Jamais le cadavre n'est laissé seul. Parfois des femmes sont payées pour chanter des lamentations (tam. oppari).

Très tôt, on procède à la désignation d'un kartta. Ce terme est dérivé d'une racine sanskrite signifiant "action", "création". Tout au long du rituel funéraire qui s'étend souvent sur un mois complet, le kartta doit se rendre disponible pour présider les rituels et en garantir le bon déroulement. Tous les jours, il sera vêtu d'un vesti et d'un châle (tam. calvai) blancs. De plus, il est traditionnel à Sri Lanka pour le kartta de se faire raser la tête. Tout au long du rituel, le kartta sera l'agent, le responsable du perfectionnement de l'âme du défunt, celui qui accomplira les actes prescrits au nom de tous les endeuillés. Quiconque a connu le disparu peut être désigné kartta, mais il existe une liste hiérarchique en tête de laquelle on trouve les fils, puis le conjoint, les filles, les frères, etc. Les fils demeurent toujours le premier choix, l'aîné pour un père et le cadet pour sa mère.

Le jour de la crémation, une importante cérémonie en trois temps se tient d'abord à l'extérieur de la maison du défunt. Le cercueil ouvert est installé dans la cour sur des tréteaux et un prêtre procède à la sanctification de l'âme du défunt au moyen d'une 
oblation au feu (skt. homa). Les parents du défunt, d'abord les hommes, s'approchent pour appliquer à tour de rôle un peu d'huile de sésame sur le front du défunt. Il arrive qu'un assistant tienne un miroir au-dessus du cercueil au bénéfice, explique-t-on, de l'âme du défunt réputée se tenir alors à l'extérieur du corps, observant la cérémonie.

Certains salons funéraires mettent toutefois à la disposition des proches une salle pour la toilette rituelle du défunt et la préparation du corps en vue de son exposition.
On chante ensuite l'hymne Tirupporcunnam ("Poussière d'or sacrée"), composé au IX" siècle par le poète tamoul Manikkav acakar.Tandis que les proches entourent le cercueil, un flambeau à la main, le kartta, près du foyer sacrificiel, tient un grand pilon (tam. ulakkai). Au fond d'un mortier en bois (tam. ural), on a déposé du curcuma et de l'huile de sésame et, après chaque couplet de l'hymne triste, le kartta donne un coup dans le mortier. À la fin du chant, il recueille un peu de cette pâte et l'applique au front du défunt.

Puis, avant que les Koviyar ${ }^{(13)}$ n'emportent le corps au terrain de crémation, les femmes font à leur tour leurs adieux au défunt. La dernière à s'exécuter est l'épouse du défunt. Elle retire alors son collier de mariage ( $\operatorname{tam}$. tali) et le dépose entre les mains jointes de son mari. Le cortège funèbre se met ensuite en branle. Tout au long du parcours, le blanchisseur de la famille fait étendre des laizes de coton blanc. Au fur et à mesure qu'on avance, les laizes sont récupérées à l'arrière puis ramenées à l'avant pour y être à nouveau étendues. Pendant ce temps, à la maison du défunt, on procède à un grand nettoyage. Près de la barrière d'entrée, on place un seau d'eau et le grand pilon est déposé en travers du seuil.

Voyons maintenant quelques-unes des modifications qui ont été apportées à ce rituel depuis l'établissement en sol québécois de la communauté tamoule originaire de Sri Lanka. Au chapitre de ce qui ne se fait plus, mentionnons l'intervention des gens de caste tels que Koviyar, barbiers, blanchisseurs et crémateurs. La loi québécoise impose qu'on retienne les services d'un directeur de funérailles et l'embaumement des corps est systématique. Les gens décédant le plus souvent à l'hôpital, c'est l'entreprise de pompes funèbres qui prend en charge le cadavre. Certains salons funéraires mettent toutefois à la disposition des proches une salle pour la toilette rituelle du défunt et la préparation du corps en vue de son exposition.

Même si le corps n'y est pas présent, on continue de se rendre à la maison endeuillée pour présenter ses condoléances, mais on n'embauche plus d'oppari pour pleurer le mort. Le rituel de sanctification du défunt a toujours lieu, mais il se déroule désormais au salon funéraire, avant la crémation. Le prêtre officiant délimite une aire sacrée près du cercueil et y dépose un grand drap blanc. Il y déploie tous ses accessoires et 
les ingrédients nécessaires à l'oblation par le feu : foyer, vaisseaux, noix de coco, beurre clarifié, etc. Dans quelques complexes funéraires, un puissant système de circulation d'air a été installé, permettant de brûler du camphre, des herbes et de l'encens.

Au salon, les visiteurs sont nombreux à ne plus se déchausser, particulièrement les plus jeunes. Souvent, même le défunt, dans son cercueil, est chaussé. L'application d'huile sur le front est maintenue, de même que l'usage du miroir. Parents et amis déposent des guirlandes dans le cercueil et du riz jaune sur la bouche du défunt. Les pièces de monnaie sur les paupières ont été remplacées par des pétales de fleur, et la préséance des hommes n'est plus respectée. L'hymne Tirupporcunnam est encore chanté à l'occasion et, après chaque couplet, le kartta frappe du pilon dans un mortier, tandis que les proches du défunt se tiennent autour du cercueil, avec à la main un bâton d'encens ou un feu de Bengale plutôt qu'un flambeau. Le plus souvent, le kartta ne se fait plus raser la tête, mais il porte tout de même l'ensemble vesti-calvai. Autre modification, il arrive que les kartta soient deux ou même davantage à se répartir les tâches.

\section{La crémation du corps, la collecte et la dispersion des cendres}

À Sri Lanka, les terrains de crémation sont d'ordinaire situés près d'un cours d'eau, aux limites de la ville, et la procession funèbre est souvent lente. À l'arrivée du cortège, les porteurs tournent trois fois autour du bûcher avant d'y déposer le corps. La responsabilité d'embraser celui-ci incombe au kartta, qui s'exécute de dos sans regarder, avant de quitter les lieux sans se retourner. Tous font ensuite comme lui, en laissant au crémateur le soin de terminer l'incinération. De retour à la maison, deux gestes de réintégration attendent les participants. Chacun s'asperge d'un peu d'eau tirée du seau placé à l'entrée, puis franchit un seuil symbolique en passant par-dessus le pilon déposé au sol.

Les braises refroidies, le kartta revient procéder à la collecte des restes. Aux cendres, il ajoute quelques morceaux d'os et place le tout dans deux récipients de terre cuite, un plus gros et un petit. Il pénètre ensuite dans l'eau jusqu'à mi-corps et laisse tomber par-dessus son épaule le principal pot de cendres. Le petit pot de restes est ramené à la maison du défunt et conservé à l'extérieur du foyer jusqu'au terme du deuil.

À Montréal, l'étape du cortège est souvent brève puisqu'on passe du salon d'exposition au four crématoire souvent en quelques minutes, parfois à l'intérieur du même bâtiment, et les porteurs autrefois rémunérés ont été remplacés par des parents et des amis du disparu. La tradition d'étendre des laizes de linge blanc au sol est souvent reprise, comme celle de lancer sur le cercueil des pétales de fleurs au passage du cortège. La coutume de porter le cercueil trois fois autour du bûcher est conservée, sauf qu'ici les porteurs font tourner trois fois le cercueil devant la porte du four crématoire. 
La loi québécoise rend obligatoire l'utilisation d'un cercueil pour toutes les étapes du rituel funéraire. Toutefois, à la demande de familles qui ne souhaitent pas que les matériaux du cercueil soient mêlés aux restes du défunt, la dépouille peut être transférée dans un sarcophage de carton avant d'être introduite dans le four. Le kartta n'allume plus le bûcher avec un brandon: tout est électronique. Certains crématoriums permettent toutefois au kartta d'actionner l'allumage du four. Parfois aussi, alors qu'un tapis roulant fait lentement pénétrer le cercueil dans le four, le kartta dépose sur celuici une pastille de camphre allumée. La tradition de se purifier en quittant le terrain de crémation est maintenue bien que différemment : dans le stationnement, avant de démarrer, les gens aspergent leurs vêtements et l'intérieur de leur voiture de quelques gouttelettes d'eau en bouteille. Par contre, on ne met plus de pilon en travers du seuil des maisons endeuillées.

La collecte des cendres ne se fait plus par le kartta. Les restes sont recueillis par les employés du crématorium. Ils seront triés puis rendus à la famille, avec ou sans urne, après le règlement final de la facture. La coutume de disposer des restes du défunt en deux étapes ne paraît pas être souvent suivie. Certaines familles demandent même au salon de conserver les cendres, parfois pendant plusieurs mois, dans l'attente de se rendre éventuellement à Sri Lanka pour les répandre dans un cours d'eau. Au Québec, il n'existe actuellement aucune législation nationale interdisant la dispersion des cendres. Dans la pratique, chaque municipalité peut édicter ses règlements et un des lieux les plus usités est le port de Montréal.

\section{L'apaisement de l'âme du trépassé et la constitution d'une bonne réputation}

Le rituel tamoul traditionnel commande qu'au lendemain de la crémation, dans la maison du défunt, on fabrique une effigie de ce dernier qui sera honorée sur une base quotidienne jusqu'au trentième jour suivant son décès. Le plus souvent, on drape une chaise de divers vêtements lui ayant appartenu. On place aussi une photo de lui et divers accessoires familiers tels que montre, lunettes, etc. Chaque jour, on procède à une puja à son intention. Le trentième jour, lors de la cérémonie finale des adieux, cette effigie sera immergée avec le petit pot de cendres conservées du défunt.

De plus, le huitième jour suivant le décès, un grand banquet est servi dans la maison du défunt en son hommage. Le menu comprend tous ses plats préférés, et peut même inclure viande, tabac et alcool. Dès le moment du décès, tous les parents et les proches du défunt sont frappés d'une impureté symbolique appelée acaucam. Cette pollution rituelle frappe tous les membres de la famille, ainsi que tous les parents masculins du côté paternel, même ceux résidant à l'étranger ${ }^{(14)}$. De plus, la famille est soumise à plusieurs interdictions, dont celle de se rendre au temple, et souvent, la chambre de prière demeure fermée. 
Au Québec, le deuil strict dépasse rarement trois jours. La maison retrouve vite sa vie normale et les appareils électroniques sont rapidement réactivés. Toutefois, la maisonnée demeure endeuillée pour trente jours et l'interdiction de se rendre au temple est maintenue, parfois même pour toute une année. La tradition du banquet du huitième jour est fréquemment maintenue, mais pas autant que la cérémonie du trentième jour, et c'est souvent à cette occasion que les cendres du défunt sont dispersées.

\section{La réunion du défunt avec ses ancêtres et la disposition de ses restes}

À Sri Lanka, au trentième jour suivant le décès, près d'un cours d'eau, se tient la cérémonie ultime des adieux (tam. antiyetti). Avec des brins d'herbe sacrée (tam. taruppai), le prêtre façonne un piretam, une représentation symbolique du défunt. Après prières et puja, il incinère ce piretam puis, dans un bol, il mélange ces cendres avec celles conservées à la maison. Puis le kartta, encore une fois, fait basculer le bol par-dessus son épaule dans de l'eau courante. À Montréal, cette cérémonie est le plus souvent accomplie à la maison même du défunt, et le kartta va ensuite en verser le produit dans un cours d'eau.

\section{Le retour de la famille à la vie normale et sa réintégration dans la communauté}

Le trente et unième jour, aussi bien à Sri Lanka qu'à Montréal, se tiennent trois cérémonies successives à la maison du défunt. La première, menée par un prêtre du temple fréquenté par la famille, en est une de purification et vise à lever l'interdit qui frappe le foyer et ses occupants. La salle de prière est désormais accessible, la lampe votive est rallumée, les portraits de famille sont retournés à l'endroit.

La seconde cérémonie met en présence le kartta et deux brahmanes. L'un d'eux se voit remettre par le kartta douze offrandes (tam.tanam) à l'intention de l'âme du disparu mais qui, dans les faits, iront enrichir le foyer du brahmane. À Sri Lanka, ces dons peuvent comprendre un matelas, des sandales, un vesti, un parapluie, parfois même une vache. À Montréal, celui-ci reçoit le plus souvent de l'argent. Dès les cadeaux reçus, ce brahmane quitte la maison. Son collègue procède ensuite à la première d'une série de onze cérémonies mensuelles, les cirattam (skt. sraddha), qui consistent en des offrandes faites aux ancêtres du défunt afin que ceux-ci l'accueillent favorablement parmi eux. La tradition veut que, chaque mois de la première année suivant le décès, puis ensuite une fois l'an, au jour anniversaire de sa mort, le kartta et la famille du défunt se réunissent pour une cérémonie semblable. À Montréal, certaines familles procèdent aux cirattam chaque mois, mais il arrive aussi que celles-ci soient célébrées en une seule cérémonie qui comptera alors pour toute l'année. 
Ensuite, seulement la troisième cérémonie peut débuter : un festin végétarien est servi. Les invités sont nombreux et on remet à chacun un livret commémoratif visant à rappeler le souvenir du disparu. Celui-ci contient des photographies, une brève biographie, des éléments généalogiques, quelques prières, de même que des commentaires élogieux écrits par des proches. À Montréal, il arrive encore qu'on produise des livrets commémoratifs, mais on filme aussi les cérémonies à la caméra numérique pour produire un DVD qui sera ensuite distribué, notamment sur Internet, à ceux qui n'ont pu assister aux funérailles.

\section{Conclusion}

Au total, on a noté près d'une trentaine de modifications et d'ajustements apportés au rituel funéraire çivaïte tamoul en terre montréalaise. Les lieux et l'environnement ne sont plus les mêmes. Plusieurs façons de faire ont changé. La durée des cérémonies est souvent plus courte. Une des plus importantes évolutions touche à la participation des femmes au rituel funéraire et à leurs responsabilités. À Sri Lanka, défense leur est faite de se rendre au lieu de crémation. Ici, les femmes interviennent à toutes les étapes et il n'est pas rare que certaines accompagnent le cercueil jusqu'au four crématoire.

Malgré tous ces changements, rien de fondamental ne paraît avoir changé aux yeux des membres de la communauté. Pour bien des répondants, ce qui importe surtout est de reproduire les façons de faire des ancêtres, c'est-à-dire faire porter aux survivants la responsabilité d'assurer au défunt un passage harmonieux vers son marumai, sa prochaine existence.

Le miroir, le pilon et le mortier ont repris du service à Montréal. Le banquet du huitième jour, tout comme le deuil de trente jours sont aussi souvent respectés. Pour les répondants, il est clair que malgré plusieurs transformations, l'essentiel du rituel a été conservé, tout comme le souci de transmettre la tradition. Ce qui leur importe, c'est d'exécuter le plus fidèlement possible pour leurs parents le rituel funéraire de la même façon que ceux-ci l'avaient accompli pour leurs propres parents, tout en espérant que leurs enfants feront de même à leur tour au moment de leur mort.

Ces volontés de respect et de transmission de la tradition religieuse des ancêtres font écho aux objectifs de la construction du grand temple "agamique" de Murugan de Montréal, qui possède en outre la capacité d'ancrer dans le sol de la métropole et d'afficher dans son paysage le processus de reconstruction identitaire des Tamouls hindous d'origine sri lankaise au Canada. Malgré les différences de "pureté" rituelle qui les opposent traditionnellement dans la culture tamoule, le temple et les rites adressés aux défunts sont donc finalement réunis autour de fonctions identitaires communes dans ce contexte diasporique. 


\section{Bbliographie}

- Appadurai Arjun, Worship and Conflict under Colonial Rule: A South Indian Case, Cambridge, Cambridge University Press, 2008 (1981).

- Bastenier Albert, Qu'est-ce qu'une société ethnique?, Paris, Presses universitaires de France, 2004.

- Clothey Fred W., Ritualizing on the Boundaries. Continuity and Innovation in the Tamil Diaspora, Columbia, University of South Carolina Press, 2006.

- Clothey Fred W., The Many Faces of Murukan: The History and Meaning of a South Indian God, The Hague, Paris, New York, Mouton Publishers, 1978.

- Dayalan D., Early Temples of Tamilnadu: Their Role in Socio-Economic Life, New Delhi, Harman Publishing House, 1992.

- Elmore Mark, "Contemporary hindu approaches to death. Living with the dead", in Garces-Foley Kathleen, Death and Religion in a Changing World. Armonk, N.Y., M.E. Sharpe, 2006.

- Leach Edmund, Aspects of Caste in South India, Ceylon and North-West Pakistan, Cambridge University Press, 1979.

- MCSA (Malaysian Ceylon Saivites Association), The Final Rites of the Saivites, Kuala Lumpur, Dunia Enterprises, 2005.

- Pfaffenberger Bryan, "The cultural dimension of Tamil separatism in Sri Lanka", in Asian Survey, University of California Press, vol. 21, n 11 novembre 1981, pp. 1145-1157.

- Pillay K. K., A Social History of the Tamils, part. 1, Madras, University of Madras, 1969.

- Ram Mohan, Sri Lanka. The Fractured Island, New Delhi, Penguin Books, 1989.

- Schalk Peter, "Caivam. A religion among Tamil speaking refugees from Sri Lanka", in Refugee Survey Quarterly, vol. $26, \mathrm{n}^{\circ} 2,2007$.

- Singaravélou, "Les réseaux ethnoculturels et la mondialisation: l'exemple de la diaspora indienne", in Bart François, Lenoble-Bart Annie, Afrique des réseaux et mondialisation, Karthala-MSHA, 2003, pp.17-28.

- Sutlive Vinson H., "Identity rituals", in Encyclopedia of religious rites, rituals, and festivals, New York, Routledge, 2004.

- Thiru Murugan Temple, Devotees Guide, Montréal, La Mission Saiva du Québec, non daté.

- Trouillet Pierre-Yves, "Une géographie sociale et culturelle de l'hindouisme tamoul. Le culte de Murugan en Inde du Sud et dans la diaspora", thèse de doctorat, Bordeaux, université de Bordeaux-III, 2010.

\section{Notes}

1. Mohan Ram, Sri Lanka. The Fractured Island, New Delhi, Penguin Books, 1989, p. 53.

2. Peter Schalk, "Caivam. A religion among Tamil speaking refugees from Sri Lanka", in Refugee Survey Quarterly, vol. 26, n², 2007 .

3. Albert Bastenier, Qu'est-ce qu'une société ethnique?, Paris, Presses universitaires de France, 2004.

4. Vinson H. Sutlive, "Identity rituals", in Encyclopedia of religious rites, rituals, and festivals, New York, Routledge, 2004.

5. Fred W. Clothey, Ritualizing on the Boundaries. Continuity and Innovation in the Tamil Diaspora, Columbia, University of South Carolina Press, 2006.

6. Arjun Appadurai, Worship and Conflict under Colonial Rule. A South Indian Case, Cambridge, Cambridge University Press, 2008 (1981); D. Dayalan, Early Temples of Tamilnadu: Their Role in Socio-Economic Life, New Delhi, Harman Publishing House, 1992 ; Bryan Pfaffenberger, "The cultural dimension of Tamil separatism in Sri Lanka", in Asian Survey, University of California Press, vol. 21, n 11 november 1981, pp. 1145-1157; K. K. Pillay, A Social History of the Tamils, part. 1, Madras, University of Madras, 1969.

7. Préceptes de la religion aiva.

8. Thiru Murugan Temple, Devotees Guide, Montréal, La Mission Saiva du Québec, non daté, p. 10.

9. Clothey Fred W., The Many Faces of Murukan: The History and Meaning of a South Indian God, The Hague, Paris, New York, Mouton Publishers, 1978 ; Trouillet Pierre-Yves, "Une géographie sociale et culturelle de l'hindouisme tamoul. Le culte de Murugan en Inde du Sud et dans la diaspora", thèse de doctorat, Bordeaux, université de Bordeaux-III, 2010.

10. Thiru Murugan Temple, op.cit., p. 10.

11. Singaravélou, "Les réseaux ethnoculturels et la mondialisation: l'exemple de la diaspora indienne", in Bart François, Lenoble-Bart Annie, Afrique des réseaux et mondialisation, Karthala-MSHA, 2003, p. 28.

12. MCSA (Malaysian Ceylon Saivites Association), The Final Rites of the Saivites, Kuala Lumpur, Dunia Enterprises, 2005.

13. Les Koviyar constituaient une caste servile, associée au personnel domestique des Vellalar. Même s'ils transportaient les corps de Vellalar, ils n'étaient pas considérés comme intouchables. Voir Edmund Leach, Aspects of caste in South India, Ceylon and North-West Pakistan, Cambridge University Press, 1979.

14. Mark Elmore, "Contemporary Hindu approaches to dath. Living with the dead", in Garces-Foley Kathleen, Death and Religion in a Changing World. Armonk, N.Y., M.E. Sharpe, 2006. 